\title{
Secondary resection tracheal was facilitated in robot-assisted left pneumonectomy
}

\author{
Hongtao Duan", Liping Tong", Yifang Zhu, Xiaoping Dong, Yong Zhang, Honggang Liu, Xiaolong Yan \\ Department of Thoracic Surgery, Tangdu Hospital, Air Force Medical University, Xi'an 710038, China \\ \#These authors contributed equally to this work. \\ Correspondence to: Prof. Xiaolong Yan. Department of Thoracic Surgery, Tangdu Hospital, Air Force Medical University, 569 Xinsi road, Xi'an \\ 710038, China. Email: yanxiaolong@fmmu.edu.cn; Prof. Honggang Liu. Department of Thoracic Surgery, Tangdu Hospital, Air Force Medical \\ University, 569 Xinsi Road, Xi’an 710038, China. Email: drliugh@163.com.
}

\begin{abstract}
The purpose of the essay is to introduce a new procedure in term of robot-assisted left pneumonectomy. The "secondary resection tracheal" technique is that, the left bronchi, which was $3-4 \mathrm{~cm}$ apart from trachea carina was disserted firstly, as the distal bronchi stump was lifted, with more space and vision being explored, the artery was pinned down easily, chipped and cut accurately. As far as the program goes down, it is more privileged but one point where it was capable of avoiding or decreasing the risk of ripping the left artery. In addition to isolating, lifting or tracting the proximal tracheal stump, the endoGIA staple was much easier used to cut the tracheal stump secondly and to deliver shorter one as well as to reduce the odds of the bronchial stump fistula. Moreover, for the preliminary date has indicated, there's been a significant decrease in intraoperative hemorrhage involving with the left pneumonectomy.
\end{abstract}

Keywords: Robot-assisted surgery; left pneumonectomy; secondary resection tracheal

Submitted May 15, 2018. Accepted for publication Nov 23, 2018.

doi: $10.21037 /$ jtd.2018.11.136

View this article at: http://dx.doi.org/10.21037/jtd.2018.11.136

\section{Introduction}

Pneumonectomy was an alternative when lung-preserving surgery was not up to handle with the scenario about the lung cancer treatment. So far, minimally invasive surgery has been in full blossom with the development of the minimally invasive instruments, especially the Staplers (1). Although there have been some advantages in the Robot-Assisted surgery (2), pneumonectomy is still generally recognized with hugely hurdling in the lung surgery. Because there are not enough clinical dates to illustrate and explore technical difficulties of pneumonectomy in the robotassisted surgery, so this approach is far away from being carried out widely. According to Lorenzo Spaggiari (3), robotic pneumonectomy still is relatively hard endeavor to achieve optimal proximal bronchial transection behind the aortic arch. And as there was an increasing risk with the artery being teared during the procedure-the fact, left main tracheal and artery are surrounded by arcus aortae and the thoracic artery-the left pneumonectomy would be filled with technically challenging. So, we bring up the robot-assisted left pneumonectomy based on the secondary resection tracheal.

\section{Operative technique (Figure 1)}

The "secondary resection tracheal" technique is that, after isolating the superior and inferior pulmonary veins, we creatively dissert firstly the bronchi which was apart from 3-4 cm trachea carina, the assistant who lift or tract the distal bronchi stump can increase surgical freedom and it is safe and effective for the surgeon to divide the pulmonary artery. Accompanied by taking the left lung, the proximal tracheal stump can be the secondary resection by the Endo-GIA staple. It can achieve safely and effectively to dissert and divide the pulmonary artery, at the same time avoid excessive tracheal stump and decrease the possible of bronchial stump fistula. 


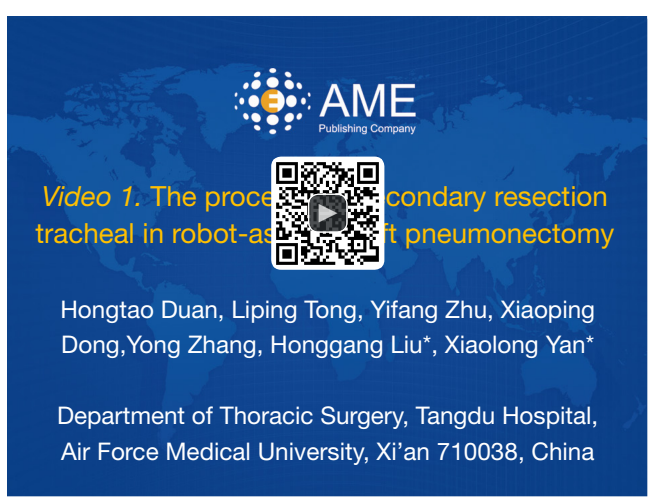

Figure 1 The procedure of econdary resection tracheal in robotassisted left pneumonectomy (4).

Available online: http://www.asvide.com/article/view/29197

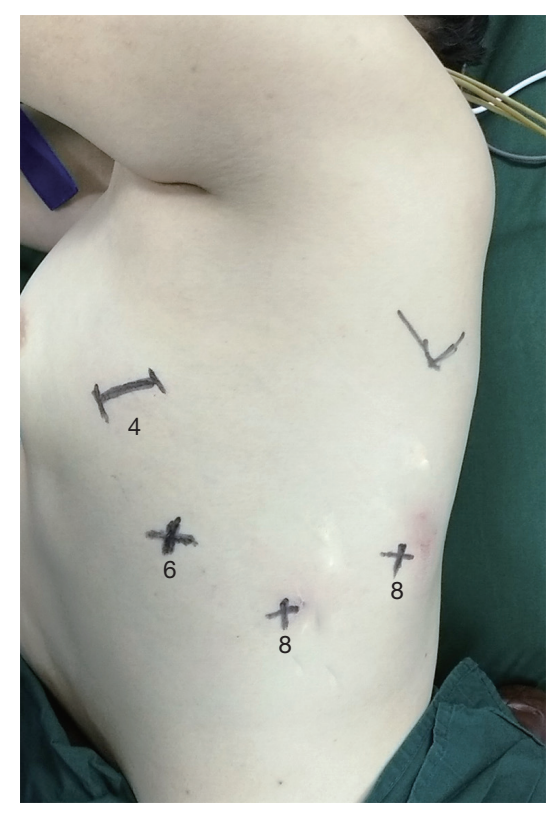

Figure 2 The incisions position of the left thorax.

A $12-\mathrm{mm}$ camera port was cut along the eighth interspace posterior axillary line. A 8-mm incision in the sixth interspace anterior axillary line was be used by robotic arms 2 (Bipolar Forceps), another 12-mm incision in the eighth intercostal space along the line between the scapular tip and posterior axillary line was performed by robotic arms 1 (Cautery Spatula or Bipolar Forceps). At last, the 4-cm minithoracotomy was played in the fourth intercostal space anteriorly (Figure 2).

The pneumonectomy was initiated by resecting the inferior pulmonary ligaments and the inferior pulmonary vein by the stapling through fourth intercostal space anteriorly. Isolating the superior pulmonary vein near to anterior hilum, stapling is performed by removing the instrument (arm 1) in the eighth intercostal space along the line between the scapular tip and posterior axillary line. As followed, the station 7 lymph nodes were disserting out (the sequence can be changed). Continuing to dissert and isolate, it can be facilitated the exposure of the main left bronchus. The main left bronchus which was apart from 3-4 cm trachea carina was firstly cut down with the stapling. Next, the distal bronchi stump was lifted, as a result of the merits of distinct exposure, and it is safe and effective for the surgeon to divide the pulmonary artery. Owing to taking off the left lung, the surgical field was increased for surgeon to operate. The left station 4 lymph nodes were incised, meanwhile, the proximal bronchial stump were lifted along with robotic arms 1, 2 (Bipolar Forceps); Endo-GIA staple immediately was accomplished to divide the bronchial stump at the bottom of the left main tracheal in ordering to cut off the bronchial stump accurately. Closed to the anterior hilum, lymph node station 5, 6 were dissected. A piece of parietal pleura was sutured to the tracheal stump by interrupted suture (4-0, Coared, Braided Silk). After irrigating with the sterile water in the chest and testing the bronchial stump by getting $30 \mathrm{cmH}_{2} \mathrm{O}$ of pressure in the airway by anesthesia, a chest tube was inserted through the 12-mm incision (the eighth interspace posterior axillary line) (Figure 3).

Secondary resection tracheal is a safe and practicable treatment for the robotic pneumonectomy. So far, by performing the methods, there were 16 patients who had been discharged on postoperative day $5-7$. This is similar to the Lorenzo Spaggiari. And there was no difference in operation time and intraoperative blood loss about ripping the left main artery compared to the VATS.

\section{Comments}

Robotic-assisted surgery has stood chances to become mainstream treatment for decades (2), especially robotic assisted pneumonectomy which has been upgrading. So far, there were different levels with application of roboticassisted pneumonectomy. Two kinds of the typical methods were used: three arms and four arms (5). Our team workers, similar to the Lorenzo Spaggiari, performed the procedure with the three arms. It requires an assistant, who must suck, do traction, lift the lung and accomplish the Staplers with vascular and bronchial transaction. Comparing to the four 

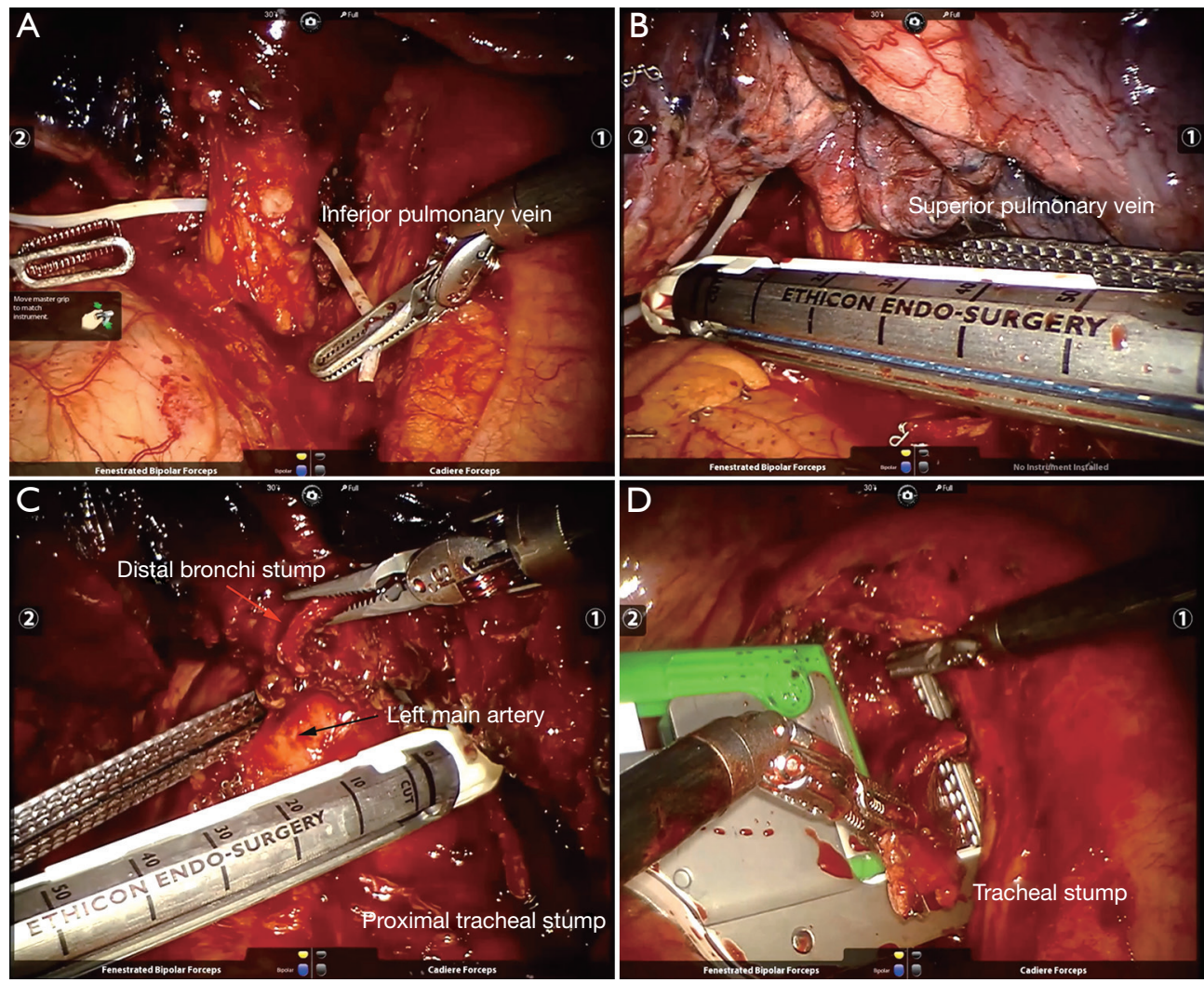

Figure 3 The "secondary resection tracheal" technique is that, after isolating the superior and inferior pulmonary veins (A,B), we creatively dissert firstly the bronchi which was apart from 3-4 cm trachea carina, the assistant who lift or tract the distal bronchi stump can increase surgical freedom (C) and it is safe and effective for the surgeon to divide the pulmonary artery (D).

arms, three arms is favorite with minimally invasive trauma.

One point set forth in the article where the robotic secondary resection tracheal procedure can avert or decrease the risk of the intraoperative hemorrhage about ripping the left artery. Rohit K. Sahai supposed that to minimize bleeding during the pneumonectomy depended on the degree of exposure (6). According to the Athar Battoo' experience of the thoracoscopic pneumonectomy (7), in order to avoid catastrophic bleeding event, they innovatively held the tracheal with $14 \mathrm{~F}$ leader catheters with the left main artery unfettered. That initially we dissert the tracheal firstly and then the distal tracheal stump will be lifted is the highly effective strategy to provide us more space and vision, so the left main artery was isolated and disserted smoothly, it's a way of ensuing not to tear apart the left artery.

Among the merits about secondary resection tracheal, it would reduce the chance of the bronchopleural fistula. Because the stump can be cut accurately and restricted as shorten as possible. At multivariate analysis (8), the length of bronchial stump was considered as the independent risk factors for the bronchi pleural fistula after pneumonectomy. In our procedure, after tracheal was cut and sutured with the staplers firstly, it would assist with exposing more horizons to operate effectively and safely, enable to isolate the bottom of the left main tracheal conveniently, and make sure Endo-GIA staple immediately divided the bronchial stump accurately.

From an oncologic standpoint, another concern was mediastinal lymphadenectomy which have been performed. In fact, en bloc resection and uncluttered to dissect the lymph node is equal to the video-assisted thoracic surgery (VATS). Compared with the VATS, both the number of lymph nodes and the number of lymph node stations removed were alike to robotic pneumonectomy in this study.

In conclusion, the salient feature of the procedure is that after initially cutting the bronchi, the distal tracheal stump 
can be lifted, as a result that it would make easier and more effective to separate and cut off the artery. At the same time, we can dissert on the bottom of left bronchi and assure to shorten the size of the stump.

\section{Acknowledgements}

None.

\section{Footnote}

Conflicts of Interest: The authors have no conflicts of interest to declare.

Informed Consent: Written informed consent was obtained from the patient for publication of this manuscript.

\section{References}

1. Conlan AA, Sandor A. Total thoracoscopic pneumonectomy: Indications and technical considerations. J Thorac Cardiovasc Surg 2003;126:2083-5.

Cite this article as: Duan $\mathrm{H}$, Tong L, Zhu Y, Dong X, Zhang Y, Liu H, Yan X. Secondary resection tracheal was facilitated in robot-assisted left pneumonectomy. J Thorac Dis 2018;10(12):7005-7008. doi: 10.21037/jtd.2018.11.136
2. Louie BE. Robotic pneumonectomy. Thorac Surg Clin 2014;24:169-75.

3. Spaggiari L, Galetta D. Pneumonectomy for lung cancer: A further step in minimally invasive surgery. Ann Thorac Surg 2011;91:e45-7.

4. Duan H, Tong L, Zhu Y, et al. The procedure of econdary resection tracheal in robot-assisted left pneumonectomy. Asvide 2018;5:935. Available online: http://www.asvide.com/ article/view/29197

5. Agzarian J, Fahim C, Shargall Y, et al. The use of Robotic-Assisted thoracic surgery for lung resection: A comprehensive systematic review. Semin Thorac Cardiovasc Surg 2016;28:182-92.

6. Sahai RK, Nwogu CE, Yendamuri S, et al. Is thoracoscopic pneumonectomy safe? Ann Thorac Surg 2009;88:1086-92.

7. Fujimoto T, Zaboura G, Fechner S, et al. Completion pneumonectomy: Current indications, complications, and results. J Thorac Cardiovasc Surg 2001;121:484-490.

8. Di Maio M, Perrone F, Deschamps C, et al. A metaanalysis of the impact of bronchial stump coverage on the risk of bronchopleural fistula after pneumonectomy. Eur J Cardiothorac Surg 2015;48:196-200. 